\title{
The effects of $\boldsymbol{N}$-alkylmaleimides on the activity of rat liver glucose 6-phosphatase
}

\author{
Behzad VAKILI and Malcolm BANNER \\ Department of Biochemistry, Chelsea College, London SW3 6LX, U.K.
}

(Received 23 June 1980/Accepted 12 September 1980)

\begin{abstract}
A series of $N$-alkylmaleimides has been synthesized and used to investigate the thiol groups that are essential for the activity of rat liver microsomal glucose 6-phosphatase. All of the $\mathrm{N}$-alkylmaleimides inactivated glucose 6-phosphatase when preincubated with microsomes (microsomal fractions) at $\mathrm{pH} 6.5$ and $30^{\circ} \mathrm{C}$. When enzyme activity was assayed in intact microsomes, the inactivation was non-linear with respect to time, showing an initial rapid phase followed by a slower secondary phase. During the initial rapid phase the inactivation may apparently be completely reversed by disrupting the microsomal membrane with detergent. However, after longer exposure to $N$ alkylmaleimides the reversal is no longer complete. This observation was explained by the results obtained from studying the inactivation in detergent-disrupted microsomes. In this case glucose 6-phosphatase was also completely inactivated, but much more slowly than was seen in intact microsomes, and the process was linear with respect to time. When assayed in both intact and detergent-disrupted microsomes, glucose 6-phosphatase inactivation was dependent on the number of carbon atoms in the alkyl side chain of the $\mathrm{N}$-alkylmaleimides; this dependence was much more marked in disrupted microsomes. Analysis of the data showed that in neither case was there a saturating effect at high concentrations of maleimide. The data have been interpreted to suggest that there are are least two thiol groups essential for activity located in two separate non-polar regions of the membrane-enzyme system. The conclusions are discussed in the light of the current model for the microsomal glucose 6-phosphatase system.
\end{abstract}

Rat liver glucose 6-phosphatase (EC 3.1.3.9) is an intrinsic part of the endoplasmic-reticulum membrane. In common with some other membraneassociated enzymes it exhibits the phenomenon of latency, that is, in freshly prepared microsomal vesicles, part of the activity is only expressed when the membrane is disrupted by detergents (e.g. Snoke \& Nordlie, 1967) or by other means. This disruption not only increases the $V_{\max }$ of the enzyme, but also decreases $K_{\mathrm{m}}$ and alters the substrate specificity (Arion et al., 1972). These changes have been rationalized in a model for the enzyme proposed by Arion et al. (1975) in which it has been suggested that the hydrolytic site of the enzyme is located on the luminal side of the microsomal vesicles. Substrate molecules are translocated to this hydrolase site by a carrier protein or 'permease'. In this system it is proposed that the hydrolase is relatively nonspecific and the 'permease' is highly specific for substrate.
A role for thiol groups in the enzyme has been implied from the inhibition of activity by a variety of reagents that react with thiol groups (Wallin \& Arion, 1972; Colilla \& Nordlie, 1973) and from the presence of a group with a $\mathrm{p} K$ value of 8.7 (Colilla \& Nordlie, 1973). It has been suggested by Wallin \& Arion (1972) that inhibition by $N$-ethylmaleimide and by 5,5'-dithiobis-(2-nitrobenzoic acid) depends on an intact microsomal membrane and, therefore, that a thiol group is possibly important for the functioning of the putative 'permease' protein.

Because of the apparent importance of thiol groups to this enzyme, we have attempted a systematic study by using a series of maleimides with increasingly hydrophobic properties to examine the nature of the site of the thiol group(s) on which the enzyme activity depends. The results of the present study indicate that there are at least two different thiol groups involved in glucose 6phosphatase activity in intact microsomes 
(microsomal fractions). One of these is dependent on an intact membrane and therefore seems to be associated with the putative permease; the other is not membrane dependent, and may be directly involved at the catalytic (phosphohydrolase) site. Both of these thiol groups are judged to be in relatively hydrophobic environments, but the catalytic thiol group is much more dependent on the hydrophobic nature of the $N$-alkylmaleimide than is the membrane-dependent (permease) thiol group, and therefore appears to be in a much more hydrophobic environment.

\section{Experimental \\ Chemicals}

$\mathrm{N}$-Ethylmaleimide and D-glucose 6-phosphate (monosodium salt) were obtained from Sigma, maleic anhydride, hexyl-1-amine and octyl-1-amine from Eastman-Kodak. All other reagents were of the highest grade available from $\mathrm{BDH}$.

\section{Synthesis of $N$-alkylmaleimides}

$N$-Butyl-, $N$-hexyl- and $N$-octyl-maleimides were synthesized from maleic anhydride and the corresponding $N$-alkylamines via the $N$-maleamic acids. The $N$-maleamic acids were made as described by Tsou et al. (1955) and converted into the $N$ alkylmaleimides as described by Heitz et al. (1968).

The $N$-alkylmaleimides were examined by i.r. and n.m.r. spectroscopy. In addition, reactivity of the compounds with cysteine was measured by using Ellman's reagent [5,5' -dithiobis-(2-nitrobenzoic acid)] to detect residual thiol groups. No evidence of significant impurities was detected.

\section{Preparation of microsomes}

Microsomes were prepared from the livers of adult male rats weighing approx. $200 \mathrm{~g}$. Animals were deprived of food overnight. They were killed by decapitation and the livers removed and homogenized in $0.25 \mathrm{M}$-sucrose $/ 1 \mathrm{mM}$ EDTA/ $/ 10 \mathrm{~mm}$ Tris, $\mathrm{pH} 7.4$, in a Potter-Elvehjem-type homogenizer fitted with a Teflon pestle. The homogenate $(20 \%, w / v)$ was centrifuged at $12000 \mathrm{~g}$ for $20 \mathrm{~min}$ at $4^{\circ} \mathrm{C}$ in an MSE $6 \times 100 \mathrm{ml}$ rotor $\left(r_{\mathrm{av}} .8 .0 \mathrm{~cm}\right)$. The pellet was resuspended in the same medium and re-centrifuged under the above conditions. The pooled supernatants were centrifuged at $105000 \mathrm{~g}$ for $60 \mathrm{~min}$ at $4^{\circ} \mathrm{C}$ in an MSE $8 \times 50 \mathrm{ml}$ rotor $\left(r_{\text {av }}\right.$. $7.5 \mathrm{~cm}$ ). The microsomal pellets were washed once in the buffered sucrose and resuspended in the same solution. Microsomal suspensions were stored at $-20^{\circ} \mathrm{C}$.

\section{Enzyme assays}

Glucose 6-phosphatase activity was measured at $30^{\circ} \mathrm{C}$ in reaction mixtures containing final concentrations of $40 \mathrm{~mm}$-sodium cacodylate adjusted to
pH6.5, 25 mM-v-glucose 6-phosphate and between 0.12 and $0.25 \mathrm{mg}$ of microsomal protein in a final volume of $1.0 \mathrm{ml}$. For the assay of detergentdisrupted microsomes, bovine serum albumin at a final concentration of $10 \mathrm{mg} / \mathrm{ml}$ was also included in incubation mixtures. Reactions were terminated with an equal volume of $10 \%(\mathrm{w} / \mathrm{v})$ trichloroacetic acid, and the $P_{1}$ in the supernatants was determined by the method of Delsal \& Manhouri (1958).

The latency of glucose 6-phosphatase activity, as defined by Arion et al. (1972), was measured by supplementing microsomal preparations with sodium deoxycholate to a final concentration of $0.26 \%(\mathrm{w} / \mathrm{v})$. The latency of glucose 6-phosphatase activity in preparations used in the present study was between 40 and $50 \%$, and mannose 6phosphatase activity $90-95 \%$. Values for nonspecific phosphatase (de Duve et al., 1949) were negligible.

\section{Inactivation by $\mathrm{N}$-alkylmaleimides}

Experiments were performed by preincubating microsomes at $30^{\circ} \mathrm{C}$ in $40 \mathrm{~mm}$-sodium cacodylate buffer, pH 6.5, with various concentrations of each of the $\mathrm{N}$-alkylmaleimides. These preincubation mixtures normally contained between 3.2 and $4.5 \mathrm{mg}$ of microsomal protein $/ \mathrm{ml}$. Reactions were terminated either by dilution of samples at least 20 -fold into the enzyme assay medium or alternatively by transferring samples to solutions of L-cysteine such that the final concentration of the L-cysteine was in at least 5 -fold excess over the $N$-alkylmaleimide concentration. The resulting solutions were kept on ice and supplemented with either sodium deoxycholate to a final concentration of $0.26 \%(\mathrm{w} / \mathrm{v})$ or with water before glucose 6-phosphatase activity was measured.

There was no observable difference in the results whichever method was used for terminating the reaction with maleimides.

\section{Results}

\section{Time-dependent inactivation by $\mathrm{N}$-alkylmaleimides}

No significant inactivation of glucose 6phosphatase activity was observed when microsomal preparations were incubated at $30^{\circ} \mathrm{C}$ and $\mathrm{pH} 6.5$ either in the presence or absence of $\mathrm{N}$-alkylamines. Hence, any inactivation observed under our conditions was not due to thermal inactivation, nor directly to the effect of the alkyl side chains of the series of reagents used in the present studies.

The time courses for the inactivation of glucose 6-phosphatase of intact microsomes by $N$-ethyl-, $N$-butyl-, $N$-hexyl-, and $N$-octyl-maleimide are shown in Fig. 1. Semilogarithmic plots are non-linear with respect to time, showing a very rapid initial fall in activity followed by a transition to a second slower rate of inactivation. Enzyme activity was 


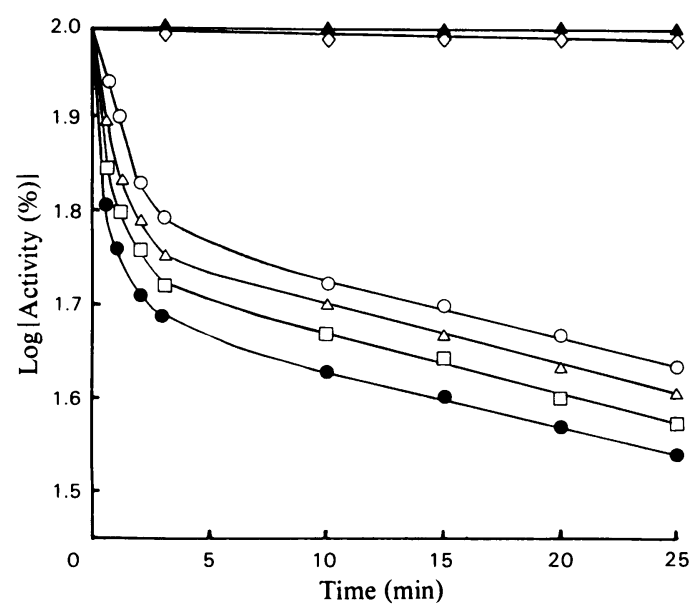

Fig. 1. Effect of $N$-alkylmaleimides on glucose 6phosphatase activity assayed in intact microsomes Preincubation mixtures contained either $0.2 \mathrm{~mm}$ $N$-ethylmaleimide (O), $0.2 \mathrm{mM}-N$-butylmaleimide $(\triangle), 0.2 \mathrm{mM}-N$-hexylmaleimide $(\square)$ or $0.2 \mathrm{mM}-N$ octylmaleimide (O). Controls were supplemented with either distilled water $(\Delta)$ or $0.2 \mathrm{mM}-\mathrm{N}$ hexylamine $(\diamond)$ in place of the $N$-alkylmaleimides.

completely destroyed by all of the $N$ alkylmaleimides. Under the conditions used in most of the experiments described in the present paper, up to $60 \%$ of activity was lost during the initial rapid phase, with the remaining activity being destroyed more slowly during the secondary phase. Both the rate and the extent of inactivation during the rapid phase varies considerably with the concentration and chain length of the $N$-alkylmaleimide, so that, at the lowest concentrations of inhibitor, the biphasic nature of the process is hardly discernible. The secondary phase was much less sensitive to these variables, and attempts to study the effect of concentration and chain length on this phase of inactivation in intact microsomes proved impossible, since as the concentration of $\mathrm{N}$-alkylmaleimides was raised, the enzyme was almost totally inactivated during the rapid phase.

Fig. 2(a) describes the effect on the initial rapid phase of inactivation of varying the concentration of $N$-butylmaleimide. The other alkylmaleimides gave a similar series of curves. The initial rates of inactivation replotted as a function of maleimide concentration showed that the inactivation is pseudofirst-order with respect to maleimide concentration. The apparent first-order rate constants, $k_{+1}$, were calculated from $k_{+1}=0.693 / t_{0.5}$, where $t_{0.5}$ is the time taken for $50 \%$ inactivation. Replots (not shown) of $\log \left(1 / t_{0.5}\right)$ against $\log$ (maleimide concentration) (Keech \& Farrant, 1968) gave straight lines

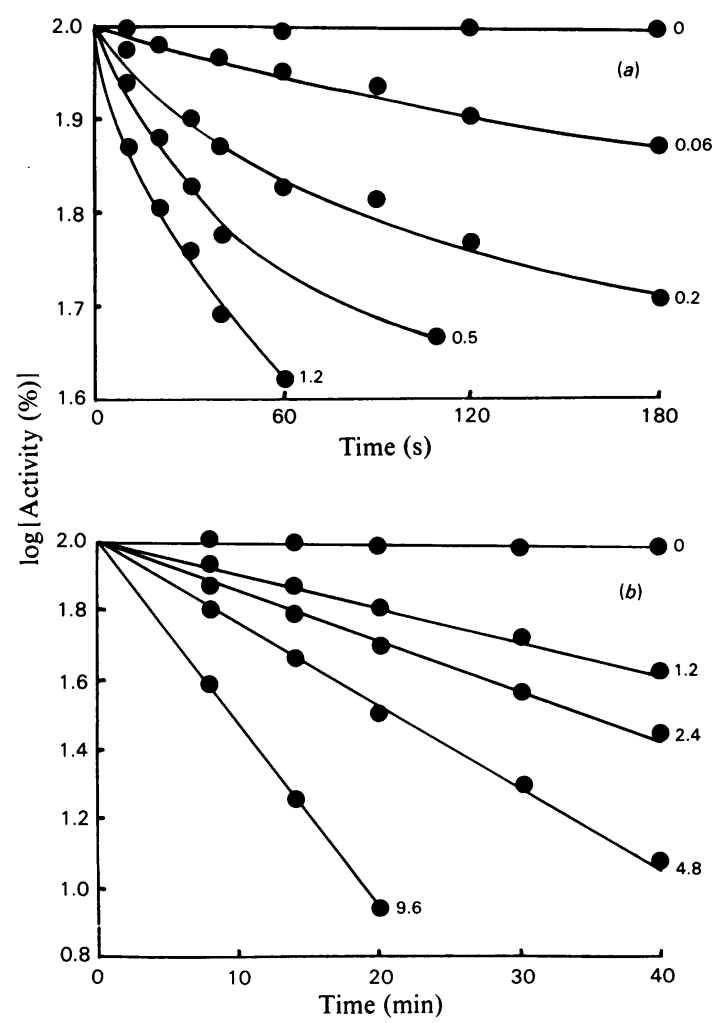

Fig. 2. Effect of various concentrations of $N$ butylmaleimide on glucose 6-phosphatase activity Preincubation mixtures contained the indicated $\mathrm{mM}$ concentrations of $N$-butylmaleimide. (a) Enzyme activity assayed in intact microsomes; $(b)$ enzyme activity assayed in detergent-disrupted microsomes.

with slopes between 1.0 and 1.2 for all $N$ alkylmaleimides, indicating that only a single thiol residue is reacting with the maleimides during this phase of inactivation. Apparent first-order rate constants were plotted against maleimide concentration (Fig. $3 a$ ), in order to determine the second-order rate constants $\left(k_{+2}\right)$ for each maleimide. Values for $k_{+2}$ increase in a linear fashion with increasing length of the side chain of the maleimide (Fig. $3 b$ ) and clearly show that the longer the chain length of the $\mathrm{N}$-alkylmaleimide the more effective it is at inactivating glucose 6phosphatase activity.

The non-linear response of enzyme activity to these reagents suggests that they are reacting with more than one thiol group. Accordingly we attempted to determine whether the different members of our series of reagents react with the same group in the enzyme. Microsomes were preincubated with a low concentration of $\mathrm{N}$-ethylmaleimide, and at various times samples were removed and transferred 

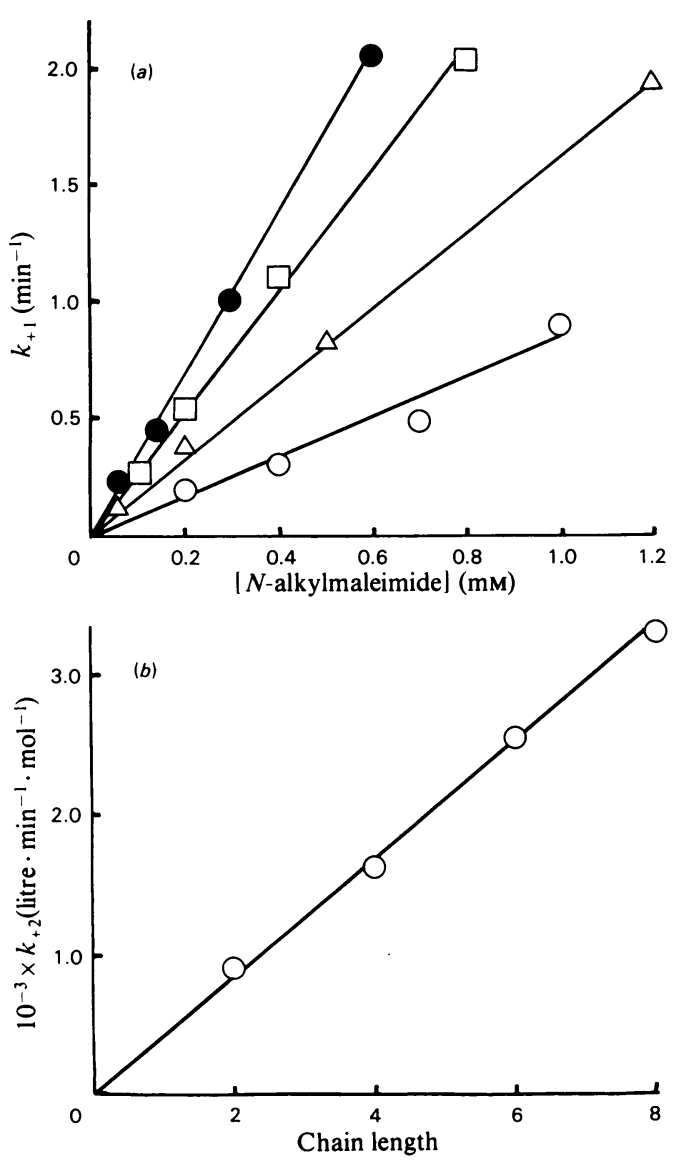

Fig. 3. Effect of chain-length of $N$-alkylmaleimides on the rate of inactivation of glucose 6-phosphatase assayed in intact microsomes

(a) Variation of pseudo-first-order rate constants with concentration of $\mathrm{N}$-ethylmaleimide (O), $\mathrm{N}$ butylmaleimide $(\triangle), N$-hexylmaleimide $(\square)$ and $N$-octylmaleimide (O). (b) Increase in the secondorder rate constants with the number of carbon atoms in the alkyl group of $\mathrm{N}$-alkylmaleimides.

to a second preincubation medium containing $N$-hexylmaleimide. The effects of both sets of preincubation on glucose 6-phosphatase activity were measured as described above. Fig. 4 shows that the extent of the rapid-phase inactivation by $N$ hexylmaleimide was decreased by the same amount as the inactivation brought about by the prior incubation with $N$-ethylmaleimide; that is, the effect of the two different maleimides was not additive. Similar results were obtained with other pairs of maleimides. It appears, therefore, that the differentchain-length maleimides react with the same groups on the enzyme, but at different rates related to the chain length of the alkyl group.

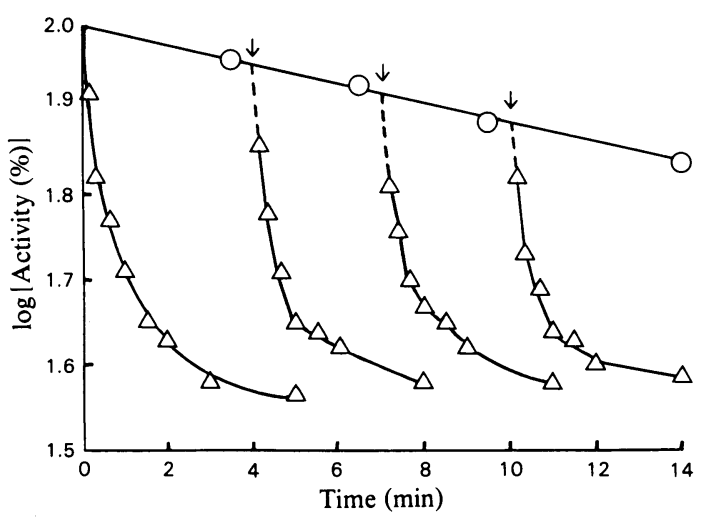

Fig. 4. Combined effects of $N$-ethylmaleimide and $N$-hexylmaleimide on glucose 6-phosphatase activity assayed in intact microsomes

Microsomes were preincubated with $0.05 \mathrm{~mm}-N$ ethylmaleimide (O) and then, at the times indicated, portions were transferred to a second preincubation mixture containing $0.6 \mathrm{~mm}-N$-hexylmaleimide $(\triangle)$.

\section{Effect of membrane disruption on the inactivation of} glucose 6-phosphatase by $\mathrm{N}$-alkylmaleimides

When microsomes were disrupted with detergent after preincubation with $\mathrm{N}$-alkylmaleimides (but before the assay of enzyme activity as described in the Experimental section), the rapid phase of inactivation was no longer apparent. This dependence of the initial rapid phase of inactivation on an intact microsomal membrane is illustrated in Fig. 5. Although intact microsomes lose $60 \%$ of their control activity during the first $60 \mathrm{~s}$ of exposure to $0.6 \mathrm{mM}-N$-hexylmaleimide, it is difficult to detect any loss in activity in disrupted microsomes during this time. Over longer periods, however, the activity in disrupted microsomes is decreased by $N$ alkylmaleimides, but with an apparent first-order rate constant some 70 -fold lower than is observed during the initial rapid-inactivation phase of intact microsomes. Furthermore, the inactivation of the enzyme measured in detergent-disrupted microsomes was completely linear at all concentrations used (Fig. 2b), indicating that the $N$-alkylmaleimides are reacting with only a single component of the enzyme system. Disruption of the microsomal membrane with detergent, which destroys the permeability barrier to substrate, therefore seems to eliminate the requirement for a thiol-dependent component observed in intact microsomes. It seems reasonable to assume that, when enzyme assays are carried out under these conditions, we are observing the effect of $\mathrm{N}$ alkylmaleimides directly on the phosphohydrolase component of the glucose 6-phosphatase system. 


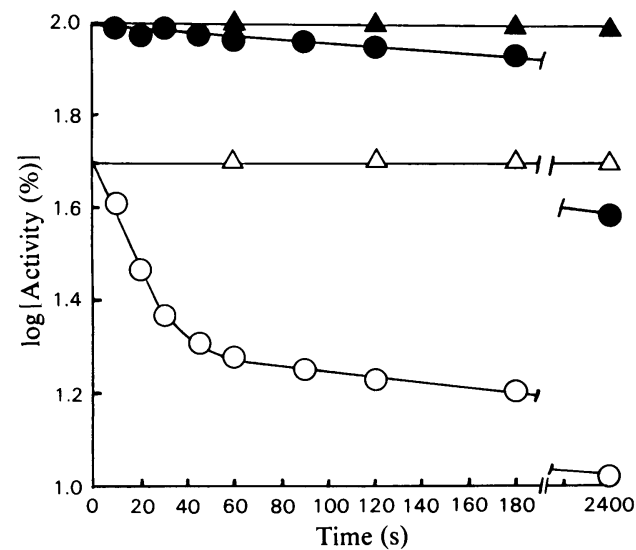

Fig. 5. Effect of deoxycholate on the inactivation of glucose 6-phosphatase by $N$-hexylmaleimide

Microsomes were preincubated with $0.6 \mathrm{mM}-N$ hexylmaleimide $(O, \boldsymbol{O})$ or water $(\Delta, \Delta)$ and then assayed either intact $(0, \Delta)$ or after disruption with $0.26 \%$ deoxycholate $(\mathbf{O}, \Delta)$ as described in the text.

Values for the second-order rate constants were determined and show an exponential increase with increasing chain length (Fig. 6); there is a linear relationship between the logarithm of the secondorder rate constants and the number of carbon atoms in the alkyl side chain of the maleimides. Thus there appears to be an even greater dependence on the hydrophobic character of the $\mathrm{N}$-alkylmaleimide than was seen for the initial rapid phase in intact microsomes.

\section{Protection by substrate}

The effect of the substrate (glucose 6-phosphate) on the rate of inactivation of glucose 6-phosphatase assayed in intact and in detergent-disrupted microsomes was determined. These data (Table 1) show
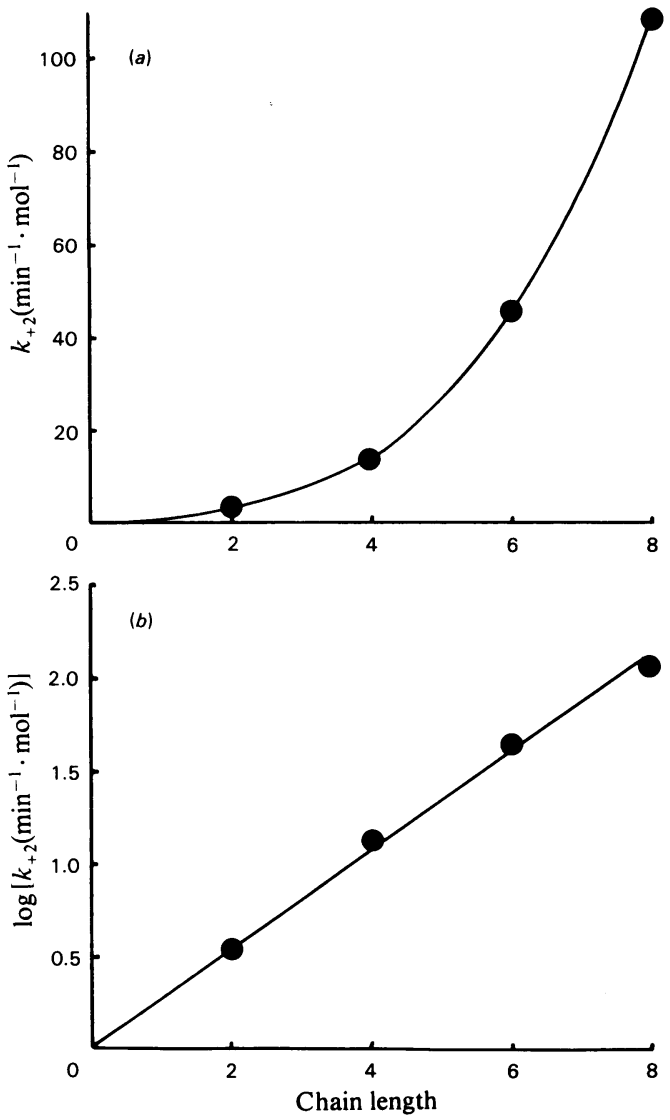

Fig. 6. Effect of various concentrations of $N$ alkylmaleimides on glucose 6-phosphatase activity assayed in deoxycholate-disrupted microsomes

Preincubation mixtures contained the indicated $\mathrm{mm}$ concentrations of $\mathrm{N}$-ethylmaleimide $(a), \mathrm{N}$-butylmaleimide $(b), N$-hexylmaleimide $(c)$ or $N$ octylmaleimide (d). After preincubation the microsomal membrane was disrupted with $0.26 \%$ deoxycholate as described in the text.

Table 1. Effect of substrate on the apparent first-order rate constants for the inactivation of glucose 6-phosphatase by $\mathrm{N}$-alkylmaleimides

Microsomes were preincubated with $N$-alkylmaleimides at $\mathrm{pH} 6.5$ and $30^{\circ} \mathrm{C}$ in the presence or absence of $20 \mathrm{mM}$ glucose 6-phosphate (Glc 6-P). Portions were removed at various times and assayed for enzyme activity both before and after disruption of the microsomes with a final concentration of $0.26 \%(w / v)$ sodium deoxycholate.

\begin{tabular}{|c|c|c|c|c|c|c|c|}
\hline \multirow[b]{3}{*}{ Maleimide } & \multirow[b]{3}{*}{ Concn. (mM) } & \multicolumn{3}{|c|}{ Without deoxycholate (initial phase) } & \multicolumn{3}{|c|}{ +Deoxycholate } \\
\hline & & \multicolumn{2}{|c|}{$k_{+1}\left(\min ^{-1}\right)$} & \multirow{2}{*}{$\begin{array}{l}\text { Decrease } \\
(\%)\end{array}$} & \multicolumn{2}{|c|}{$10^{2} \times k_{+1}\left(\min ^{-1}\right)$} & \multirow{2}{*}{$\begin{array}{l}\text { Decrease } \\
(\%)\end{array}$} \\
\hline & & -Glc 6-P & + Glc 6-P & & -Glc 6-P & + Glc $6-P$ & \\
\hline \multirow[t]{2}{*}{$N$-Ethyl } & 1.0 & $0.91 \pm 0.10$ & $0.53 \pm 0.06$ & 42 & - & - & - \\
\hline & 10.0 & $=$ & - & - & $3.50 \pm 0.09$ & $1.70 \pm 0.14$ & 51 \\
\hline \multirow{2}{*}{$N$-Butyl } & 0.5 & $0.85 \pm 0.07$ & $0.41 \pm 0.02$ & 52 & - & - & - \\
\hline & 2.4 & 二- & $=$ & - & $3.19 \pm 0.31$ & $1.88 \pm 0.04$ & 41 \\
\hline \multirow{2}{*}{$N$-Hexyl } & 0.4 & $1.12 \pm 0.09$ & $0.58 \pm 0.04$ & 48 & 二 & 二 & - \\
\hline & 0.6 & - & - & - & $2.79 \pm 0.23$ & $1.49 \pm 0.14$ & 47 \\
\hline \multirow[t]{2}{*}{$N$-Octyl } & 0.3 & $1.11 \pm 0.12$ & $0.46 \pm 0.01$ & 58 & 二 & - & - \\
\hline & 0.2 & 二 & 二 & - & $2.70 \pm 0.14$ & $1.11 \pm 0.03$ & 62 \\
\hline
\end{tabular}

Vol. 194 
that the presence of substrate in the preincubation decreased the rate of inactivation by all four maleimide derivatives by approx. 50\%. The degree of protection was similar for the rapid initial phase in intact microsomes and for the slow phase in deoxycholate-disrupted microsomes. The protection by substrate indicates that both the fast- and slow-reacting thiol groups are at, or close to, sites of substrate binding. Protection by $\mathbf{P}_{1}$ was also observed in separate experiments (results not shown), and since in the experiments on substrate protection in deoxycholate-disrupted microsomes there was considerable hydrolysis of substrate, it was not possible to distinguish between the protection afforded by glucose 6-phosphate and $\mathbf{P}_{\mathbf{i}}$.

\section{Discussion}

In the present paper we have shown that $\mathrm{N}$-alkylmaleimides can completely inactivate glucose 6-phosphatase of both intact and detergentdisrupted microsomes from rat liver. However, there are marked differences in the rates of inactivation seen under these different conditions. The extremely rapid inactivation of the enzyme in intact microsomes is dependent on the integrity of the microsomal membrane, whereas the activity is destroyed much more slowly in disrupted microsomes. Thus, when intact microsomes are exposed to $\mathrm{N}$-alkylmaleimides for short periods (on average less than $3 \mathrm{~min}$ under our conditions), the inactivation can be completely reversed by detergent. This finding provides some support for the existence of a thiol-containing, membrane-dependent component of glucose 6-phosphatase, which presumably represents the permease function of the system (Arion et al. 1975). Over longer periods of exposure of the microsomes to $\mathrm{N}$-alkylmaleimides, complete reversal is impossible, since some permanent inactivation will have occurred. This slower, irreversible inactivation of a component, which we presume to be the phosphohydrolase, is extremely dependent on the chain length of the $N$-alkylmaleimide. The value for the second-order rate constant for the inactivation by $N$-octylmaleimide was more than 50 -fold greater than the corresponding value for $N$-ethylmaleimide. The apparent reversal by detergents of the early stages of inactivation of glucose 6phosphatase activity of intact microsomes supports in general terms the permease hypothesis (Arion et al., 1975). However, it might be expected that if the rapid-phase inactivation is due to the maleimides blocking the function of a permease that is essential to enzyme activity, then virtually all the activity should be destroyed during this phase; in fact we have observed a biphasic response with a variable extent of rapid inactivation (Figs. 1 and $2 a$ ). This biphasic response could mean either that glucose 6-phosphatase hydrolysis in intact microsomes is not absolutely dependent on the function of a permease, or that membrane integrity is adversely affected during the incubation with $N$ alkylmaleimides. This latter conjecture is stimulated by the similarity of the rates of inactivation in disrupted microsomes and the slow secondary phase in intact microsomes. Our preliminary investigations into the effect of $\mathrm{N}$-alkylmaleimides on the permeability of microsomal vesicles indicate that the latency of mannose 6-phosphatase activity, normally greater than $95 \%$, decreases, whereas that of glucose 6-phosphatase activity increases until a common value is reached. This effect requires further investigation, but it does seem that $N$-alkylmaleimides increase membrane permeability. Thus, although it has not been possible to compare rate constants for the two processes for the reasons stated above, it appears likely that both represent the reaction of the same 'membrane-independent' thiol group.

The rate of inactivation in both intact and detergent-disrupted microsomes is dependent on the chain length (lipid-solubility) of the $N$ alkylmaleimide. In the case of the rapid inactivation in intact microsomes the second-order rate constants $\left(k_{+2}\right)$ increase in a linear manner with the increasing number of carbon atoms in the side chain of the maleimide used (Fig. 4). In detergent-disrupted microsomes there is also a relationship between $k_{+2}$ for the inactivation and chain length of the maleimide, but in this case there is an exponential increase in the rate of inactivation with chain length (Fig. 7).

The effects of increasing hydrophobicity of inhibitors have been observed with other enzymes (Heitz et al., 1968; Fonda \& Anderson, 1969) and have been discussed by Heitz et al. (1968). A possible explanation of this effect is that enhancement of the rate of inactivation is achieved through the formation of an initial non-covalent complex stabilized by non-polar interactions, followed by the irreversible alkylation step. We did not, however, observe any saturating effect at the highest maleimide concentrations, and double-reciprocal plots of $k_{+1}$ against maleimide concentration extrapolate to infinitely high values for $k_{+1}$ for all maleimides tested in both intact and disrupted microsomes. This finding diminishes the possibility of a non-covalent intermediate stabilized by nonpolar interactions. The most probable explanation, therefore, of the chain-length effect we have observed is that the thiol groups required for activity in both intact and disrupted microsomes are in hydrophobic environments, with the more hydrophobic inhibitors gaining more rapid access to these hydrophobic regions. Furthermore, although the range of compounds we have used gave marked differences in $k_{+2}$ values for intact and detergent- 
disrupted microsomes, extrapolation of our data suggests that these differences may at least in part be due to the differences in the hydrophobic nature of the environment of the essential groups on the enzyme, since values for $k_{+2}$ obtained with disrupted microsomes would begin to approach those obtained with intact microsomes as the chain length of the alkylmaleimides increases. In disrupted microsomes this very rapid increase with the increasing hydrophobic nature of the alkylating reagent may be interpreted as indicating that the thiol group on which enzyme activity in disrupted microsomes depends is in an extremely hydrophobic environment, more so even than the environment of the thiol group concerned in the rapid phase of inactivation seen in intact microsomes. The alkylation of both thiol groups is protected to a considerable extent by substrate, indicating that both are at, or close to, sites of substrate binding, properties that are expected of both permease and phosphohydrolase components.

Since, in our experiments, inactivation is carried out in intact microsomes followed by assay in disrupted microsomes, the increased hydrophobicity of the environment of the phosphohydrolase thiol group may simply reflect a position either buried deeply within the membrane or on the luminal face of the microsomal vesicles.

To date there is little detailed evidence concerning the nature of the two components that appear to make up the glucose 6-phosphatase system; indeed, it is not at all clear whether they are the same or different proteins. Our data provides some additional information concerning this key enzyme. Both components possess an essential thiol group, each located in a hydrophobic environment, but differ in the degree of the hydrophobicity of their environment. It seems possible that the biphasic response to maleimide inhibition that we observed in intact microsomes may reflect altered functions of the same or similar proteins related perhaps to their relative positions in the microsomal membrane.

\section{References}

Arion, W. J., Wallin, B. K., Carlson, P. W. \& Lange, A. J. (1972) J. Biol. Chem. 247, 2558-2565

Arion, W. J., Wallin, B. K., Lange, A. J. \& Ballas, L. M. (1975) Mol. Cell. Biochem. 6, 75-83

Colilla, W. \& Nordlie, R. C. (1973) Biochim. Biophys. Acta 309, 328-338

de Duve, C., Berthet, J., Hers, H. G. \& Dupret, L. (1949) Bull. Soc. Chim. Biol. 31, 1242-1253

Delsal, J. L. \& Manhouri, H. (1958) Bull. Soc. Chim. Biol. 40, 1623-1636

Fonda, M. L. \& Anderson, B. M. (1969) J. Biol. Chem. 244, 666-674

Heitz, J. R., Anderson, C. D. \& Anderson, B. M. (1968) Arch. Biochem. Biophys. 127, 627-636

Keech, D. B. \& Farrant, R. K. (1968) Biochim. Biophys. Acta 151, 493-503

Snoke, R. E. \& Nordlie, R. C. (1967) Biochim. Biophys. Acta 139, 190-192

Tsou, K. C., Barrnett, R. J. \& Seligmann, A. M. (1955) J. Am. Chem. Soc. 77, 4613-4617

Wallin, B. K. \& Arion, W. J. (1972) Biochem. Biophys. Res. Commun. 48, 694-699 